\title{
Human photopic ON- and OFF-ERG responses elicited by square wave and sawtooth stimuli
}

\author{
Gobinda Pangeni ${ }^{1}$ and Jan Kremers ${ }^{1,2}$ \\ 1- University of Erlangen-Nürnberg, Erlangen, BY, Germany \\ 2- University of Bradford, Bradford, WYK, United Kingdom
}

\begin{abstract}
In this study, the on- and off-responses elicited by luminance square wave and sawtooth stimuli at different temporal frequencies and contrasts are described quantitatively. Adding on- and off-responses reveals response asymmetries. Full-field stimuli were produced using a Ganzfeld bowl with arrays of light-emitting diodes (LEDs) as light sources. ERG responses were recorded from six normal subjects. The amplitudes and implicit times of components of the on- and off-responses and the additions were analyzed. The amplitudes of the on-, off- and addition components elicited by square wave stimuli did not depend on temporal frequency with the exception of the late negative components, which decreased with increasing temporal frequency up to $4 \mathrm{~Hz}$. The amplitudes of all components elicited by sawtooth stimuli, except the P-on, decreased with increasing temporal frequency. The amplitude of all components elicited by square wave and sawtooth stimuli were positively correlated with stimulus contrast. The implicit times of the on-components to square wave stimuli and all response components to sawtooth stimuli decreased with increasing temporal frequency. Contrast had an effect on the implicit times of the N-on, P-on, P-off and P-add components elicited by square wave stimuli and the N-on, P-on, P-add and LN-on components elicited by sawtooth stimuli. The asymmetries between on- and off-responses can possibly be used to reveal inner retinal contributions and may therefore be interesting in detecting glaucomatous changes. Keywords: electroretinogram, sawtooth stimuli, square wave stimuli, temporal frequency, contrast.
\end{abstract}

Received 31 January 2012; received in revised form 26 May 2012; accepted 27 June 2012. Available online 18 November 2013.

\section{Introduction}

Electroretinography (ERG) is a noninvasive assessment of the electrical activity of different retinal cells. Light stimuli with different spatial and temporal characteristics can be used to interpret the cellular origins of complex ERG response waveforms. ERG responses to light increments and decrements elicit distinct on- and off-responses indicating separate on- and off-pathways in the retina (Biersdorf, 1968; Howarth, 1961; Sieving, 1993). Full-field ERGs to short duration flashes have a complex waveform in which the on-response and the off-response are merged. Longer flashes elicit separate on- and off- responses (Evers \& Gouras, 1986; Kondo \& Miyake, 2000; Sieving, 1993; Sieving, Murayama \& Naarendorp, 1994; Sustar, Hawlina \& Brecelj, 2006). Alternatively, temporally modulated periodic stimuli like square wave or sawtooth stimuli may also be used to study the on-

Gobinda Pangeni and Jan Kremers, Department of Ophthalmology, University Hospital Erlangen, Germany. Jan Kremers, School of Life Sciences, University of Bradford, Bradford, UK. Correspondence regarding this article should be directed to Dr. Jan Kremers, Department of Ophthalmology, University of Erlangen-Nürnberg, Schwabachanlage 6, 91054 Erlangen, Germany. Phone: +49 9131 8544595. E-mail: jan. kremers@uk-erlangen.de and off-responses separately (Alexander, Barnes \& Fishman, 2003; Alexander, Fishman, Barnes \& Grover, 2001; Barnes, Alexander \& Fishman, 2002; Dryja et al., 2005; Khan et al., 2005; Simpson \& Viswanathan, 2007; Viswanathan, Frishman \& Robson, 2000). Periodic stimuli produce less discomfort than flashes of high intensity and owing to continuous averaging, periodic stimuli may produce a larger signal-to-noise ratio than flashed stimuli in a similar measuring time (Alexander et al., 2003; Viswanathan, Frishman, Robson, Harwerth $\&$ Smith 3rd, 1999). A change in stimulus strength and/ or temporal frequency is accompanied by a change in the state of adaptation when using flashes. With periodic stimuli these parameters can be varied without changing the mean luminance. Thus, periodic stimuli may be better suited to study on- and off-responses.

The on-responses elicited by square wave or sawtooth stimuli show an early negative wave similar to the a-wave of the flash ERG that originates in cone photoreceptors and off-bipolar cells (Bush \& Sieving, 1996; Ueno et al., 2006). The initial negative trough in the on-response is followed by a large positive deflection similar to the b-wave of the flash ERG originating in on-bipolar cells (Knapp \& Schiller, 1984; Sieving, 1993) but also in the off-bipolar and horizontal cells (Sieving et al., 1994). The off-responses display an initial positive d-wave. Pharmacological studies have 
shown that it originates in off-bipolar cell and cone photoreceptor activity (Sieving et al., 1994; Ueno et al., 2006). In both on- and off-responses, a slow negative trough homologous to the photopic negative response (PhNR) in the flash ERG is present. The PhNR was first identified in monkey flash ERGs (Viswanathan et al., 1999). This component of flash ERG was strongly reduced by intravitreal injection of tetrodotoxin (TTX), which suppresses the spiking activity of ganglion cells and amacrine cells (Bloomfield, 1996; Stafford \& Dacey, 1997) indicating that the PhNR originates in the inner retina (Viswanathan et al., 1999).

The on- and off-responses are not mirror images of each other. Mirror-imaged responses to rapid-on and rapid-off sawtooth and to the on- and offset of square wave stimuli can be expected when the signal pathway is linear or includes simple contrast-dependent nonlinearities. More complex signal pathways containing substantial nonlinearities will lead to response asymmetries between on- and off-responses. Linear responses presumably originate in the outer retina (because once nonlinearity occurs it probably will influence the signals in subsequent, more proximal stages). The response asymmetries can be extracted by adding the on- and off-responses. Linear (mirror imaged) response components will cancel each other out and the response asymmetries, possibly with a more inner retinal origin, remain. Although the on- and offresponses in the ERGs have been studied extensively (Alexander et al., 2001, 2003; Barnes et al., 2002; Khan et al., 2005; Sieving, 1993), their asymmetries have only been subject of a few studies (Rodrigues, Filho Mda, Silveira \& Kremers, 2010; Simpson \& Viswanathan, 2007; Viswanathan et al., 2000). In addition, how the response asymmetries depend on temporal frequency and stimulus strength has not yet been studied (expressed in Michelson contrast). It is the purpose of the current investigation to describe the response characteristics of the on- and off-responses as well as their asymmetries as a function of temporal frequency and contrast using periodic stimuli.

We would like to draw the attention of the reader to the fact that isolation of response asymmetries by cancellation of mirror imaged on- and off-response components is also achieved in the pattern ERG (PERG) (Baker \& Hess, 1984; Riemslag, Ringo, Spekreijse \& Verduyn Lunel, 1985; Spekreijse, van der Tweel \& Zuidema, 1973). Therefore, the response asymmetries to square wave and sawtooth stimuli are possibly an indicator of inner retinal integrity similar to the PERG (Bach \& Hoffmann, 2008; Bach et al., 2006; North, Jones, Drasdo, Wild \& Morgan, 2010). In fact, Simpson \& Viswanathan (2007) and Viswanathan et al. (2000) used the response asymmetries to square wave stimuli to simulate the PERG. However, in the PERG recordings, the stimuli have a spatial content, whereas the response asymmetries in square wave and sawtooth stimuli are spatially homogeneous. A further purpose of the current study is to compare the characteristics of the response asymmetries with those of the PERG data from the literature. Finally, if the response asymmetries originate to a large extent in the inner retina, then the data obtained in the present investigation can be used to choose optimal stimulus conditions for studying glaucomatous changes.

\section{Methods}

\section{Subjects}

ERGs were recorded from six normal subjects ranging in age between 23 and 50 years (mean \pm SD: $32.5 \pm 9.8$ ). All subjects underwent a complete ophthalmic examination and had best corrected visual acuity $20 / 20$ or better in the tested eye with clear ocular media, normal fundus and normal color vision. Informed written consent was obtained from each subject. The experiments adhered to the tenets of the Declaration of Helsinki and the protocol was approved by the institutional ethics committee.

ERGs were recorded from the right eye of each subject using Ganzfeld stimuli. The left eye was covered by a patch. A small red fixation point in the center of the Ganzfeld stimulator was used to ensure the stable fixation. The pupil of the right eye was dilated with $0.5 \%$ tropicamide (Mydriaticum, Pharma Stulln). Conjuncain EDO (oxybuprocain hydrochloride $0.04 \%$, Dr. Mann Pharma) was used as a topical anaesthetic if requested by the subject. The forehead and ipsilateral temple were cleaned with alcohol (70\% isopropyl alcohol) and scrubbed with Nuprep abrasive skin preparing gel (D.O. Weaver and Company, USA) for skin electrode placement. Gold cup electrodes filled with electrode paste (D.O. Weaver and Company) were used as ground (on the forehead) and reference electrode (on the ipsilateral temple). The active electrode was a DTL electrode placed over the lower limbus and attached at the inner and outer canthus.

\section{Visual Stimuli, ERG Recordings and Data Acquisition}

A full-field stimulus was produced using a Ganzfeld bowl (Q450SC Roland Consult, Germany) with six different colored arrays of light-emitting diodes (LEDs) as light sources controlled by a Retiport system (Roland Consult). The mean luminance, waveform, contrast and frequency of each LED array can be varied using the Retiport software. Square wave and sawtooth (rapidon and rapid-off) full-field stimuli were generated using the white LEDs (CIE coordinates: $\mathrm{x}$ : 0.37, y: $0.42)$ only. The mean luminance was $250 \mathrm{~cd} / \mathrm{m}^{2}(4.1$ log phot. td assuming an 8-mm pupil diameter). Five different Michelson contrasts [defined as: $\mathrm{C}=\left(L_{\max }-L_{\min }\right) /$ $\left.\left(L_{\max }+L_{\min }\right)\right]$ were used: $100 \%, 75 \%, 50 \%, 25 \%$ and $0 \%$ at six different temporal frequencies $(1,2,4,8,12$ and $16 \mathrm{~Hz}$ ). The $16 \mathrm{~Hz}$ square wave responses were discarded because the on- and off-responses merged. ERG measurements at $0 \%$ contrast modulation were included to obtain estimates of noise and to calculate 
the signal-to-noise ratio (SNR) for each recording. The component amplitude obtained at higher contrasts was divided by the respective component amplitude obtained at $0 \%$ contrast to calculate the SNR.

The signal was amplified 100,000 times. The signals were band-pass filtered between 1 and 300 $\mathrm{Hz}$ and digitized at $1024 \mathrm{~Hz}$. The filter settings are appropriate to capture all components that occur within about $150 \mathrm{msec}$ after the sudden change in stimulus. Fifty $\mathrm{Hz}$ noise (from the mains) was small, making the use of a notch filter unnecessary. The responses to 40 601 -sec episodes were recorded and averaged. Before the stimuli started, the mean luminance was presented to minimize onset artifacts. To avoid residual onset artifacts, the recordings to the first two stimulus cycles were discarded.

As already mentioned in the Introduction, linear on- and off-responses are mirror images of each other. One fundamental property of a linear system is that its response (R) to the sum of two stimuli ( $\mathrm{S} 1$ and $\mathrm{S} 2$ ) equals the sum of the responses to each individual stimulus:

$$
\mathrm{R}(\mathrm{S} 1+\mathrm{S} 2)=\mathrm{R}(\mathrm{S} 1)+\mathrm{R}(\mathrm{S} 2)
$$

Thus, if the stimuli S1 and S2 are of opposite polarity (i.e., $\mathrm{S} 2=-\mathrm{S} 1$ ), the addition of the responses is zero and the two responses are mirror imaged:

$$
\begin{aligned}
& \mathrm{R}(\mathrm{S} 1)+\mathrm{R}(-\mathrm{S} 1)=\mathrm{R}(0)=0 \\
& \text { thus } \mathrm{R}(\mathrm{S} 1)=-\mathrm{R}(-\mathrm{S} 1) .
\end{aligned}
$$

We used square wave and sawtooth stimuli to provide luminance increments and decrements of equal amplitude but opposite polarity. Hence, when the responses to the luminance increments and decrements are added, the linear response and simple contrast dependent nonlinearities cancel each other and asymmetrical nonlinearities in the ERG response, which most probably originate at the postreceptoral level, are revealed.

In the recorded waveforms, a baseline was defined as the average of the first five electrical potential values (corresponding to $4.8 \mathrm{msec}$ ) after the occurrence of the luminance increment or decrement. In this period a response is absent (Rodrigues et al., 2010). To measure waveform components, maxima and minima were measured. ERG waveform components to luminance increments were defined as an initial negativity ( $\mathrm{N}$-on) followed by a peak (P-on) and a late negative responses (LN-on). In the off-responses an initial peak (P-off) was followed by a late negative response (LN-off). In Figures 1 and 2, definitions of the different components are given. The amplitude of the $\mathrm{N}$-on was defined as the potential difference between baseline and the minimum in a time window between 0 and $34 \mathrm{msec}$ after the luminance increase. The P-on amplitude was measured as the difference between the minimum of $\mathrm{N}$-on and the maximum in a time window of $19-73 \mathrm{msec}$. The P-off amplitude was measured as the potential difference between the baseline and the maximum in a time window of 19-73 msec. The amplitude of the LN-on and the LN-off were defined as the potential difference between baseline and the most negative point in a time window of 49-122 msec. For $8 \mathrm{~Hz}$ square wave and 16 $\mathrm{Hz}$ sawtooth responses, one complete cycle of on- or off-responses lasted only $62.5 \mathrm{msec}$. We therefore used a time window for the b-wave and d-waves of 20-49 msec, still correctly capturing the component. For the $8 \mathrm{~Hz}$ square wave (see Figure 1) and for the $16 \mathrm{~Hz}$ sawtooth stimuli (not shown in the figure), the LN-on or LN-off components were truncated. We therefore did not include LN-on and LN-off data for these conditions. We carefully checked whether the maxima and minima were indeed captured in the respective time windows. For none of the stimulus conditions, a further adjustment of the time window (e.g., with stimulus contrast) was necessary. The time elapsed from the sudden luminance change in the stimulus to the maxima or minima of each component waveform were taken as the component's implicit time. Implicit time measurements were disregarded when the SNR (as described above) was smaller than two.

\section{Statistical Analysis}

Statistical analyses on the effects of temporal frequency and contrast on amplitudes and implicit times of different components were performed using general linear model multiple analysis of variance (MANOVA) in SPSS v.18 (SPSS Inc., Chicago, IL).

\section{Results}

Figure 1 shows examples of original responses elicited by square wave stimuli. One cycle of the square wave stimuli elicited an on- and an off-response. At $8 \mathrm{~Hz}$, the LN components were truncated. Above this frequency, the on- and off-responses merged. Figure 2 displays original responses to sawtooth stimuli. The rapid-on and rapid-off sawtooth stimuli elicited on- or off-responses, respectively. The $16 \mathrm{~Hz}$ on-and offresponses (not shown) were similar to the $8 \mathrm{~Hz}$ square wave responses and the $\mathrm{LN}$ components were truncated. The truncated LN components were not considered in the analysis. Figures 1 and 2 also display the additions of the on- and off-responses. They were large, indicating the presence of substantial asymmetries between onand off-responses. However, there was no or only a very small initial negativity indicating that the $\mathrm{N}$-on was cancelled by parts of the P-off, suggesting that these response components can be considered to be largely linear and to originate in the outer retina. This is in agreement with the literature data on the origins of their homologues in the flash ERG, the a- and d-wave (Sieving, 1993; Ueno et al., 2006).

Figures 3-6 display the mean and standard deviations (shown one-sided for clarity) of the response amplitudes (left columns) and the implicit times (right columns) from all subjects plotted as a function of temporal frequency. The results are plotted separately for different stimulus contrasts. Figures 3 and 4 show 


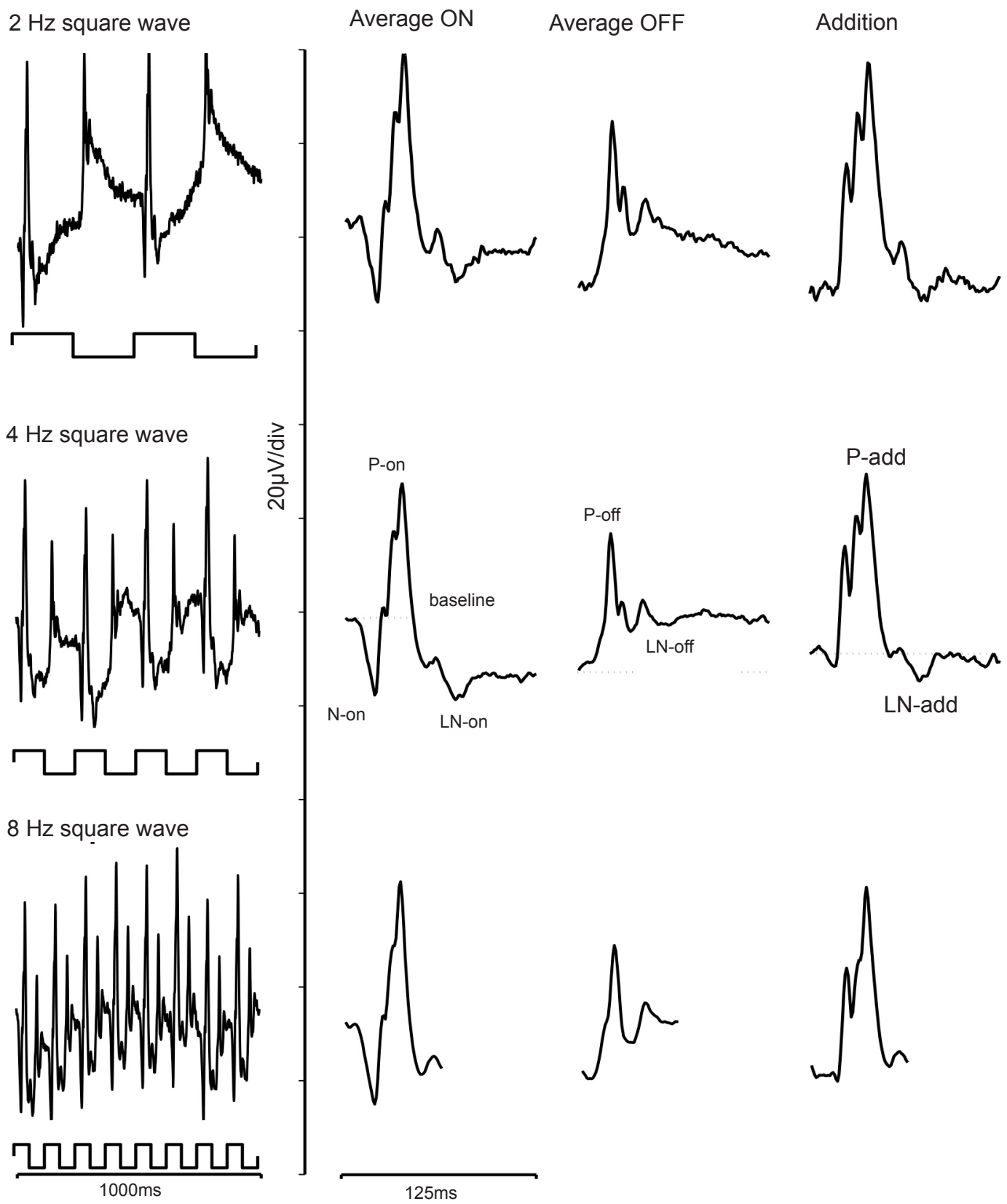

Figure 1. Examples of full-field ERGs elicited by square wave stimuli at $2 \mathrm{~Hz}$ (top), $4 \mathrm{~Hz}$ (middle) and $8 \mathrm{~Hz}$ (bottom). The left panel shows some original recordings. The responses contain on-and off-responses in a single sweep. The waveforms in the second and third columns represent the averaged on- and off-responses. The definitions of the different components ( $\mathrm{N}$-on, $\mathrm{P}$-on and the LN-on in the on-responses; the P-off and LN-off in the off-responses) are given. The right column shows the waveforms of the addition of the on-and off-responses.

the data of the measurements with the square wave stimuli, whereas Figures 5 and 6 show the sawtooth response data. Figures 3 and 5 display the data of the different on- and -off components in the responses. The amplitudes and the implicit times of the components in the response additions are shown in Figure 4 (for square wave stimuli) and Figure 6 (for sawtooth stimuli).

The MANOVA showed that temporal frequency did not have a significant effect on the amplitudes of the on-, off- and additional components elicited by square wave stimuli with the exception of the three LNs amplitudes (LN-on: $p=0.007$; LN-off: $p=0.029$;
LN-add: $p=0.003$ ) shown in the left-hand column of Figures 3 and 4. Not surprisingly, the MANOVA showed a positive relationship between amplitude and stimulus contrast for all components (N-on: $\mathrm{p}<0.001$; P-on: $\mathrm{p}<0.001$; P-off: $\mathrm{p}<0.001$; P-add: $\mathrm{p}<0.001$; LNon: $p<0.001$; LN-off: $p=0.01$; LN-add: $p=0.027$ ). There was only a measurable initial negativity in the response additions ( $\mathrm{N}$-add) at $100 \%$ contrast where its amplitude was about half the size of the N-on component. The LNon generally was larger than zero, indicating that this component is below the baseline level. The LN-off to square wave stimuli were generally above the baseline 


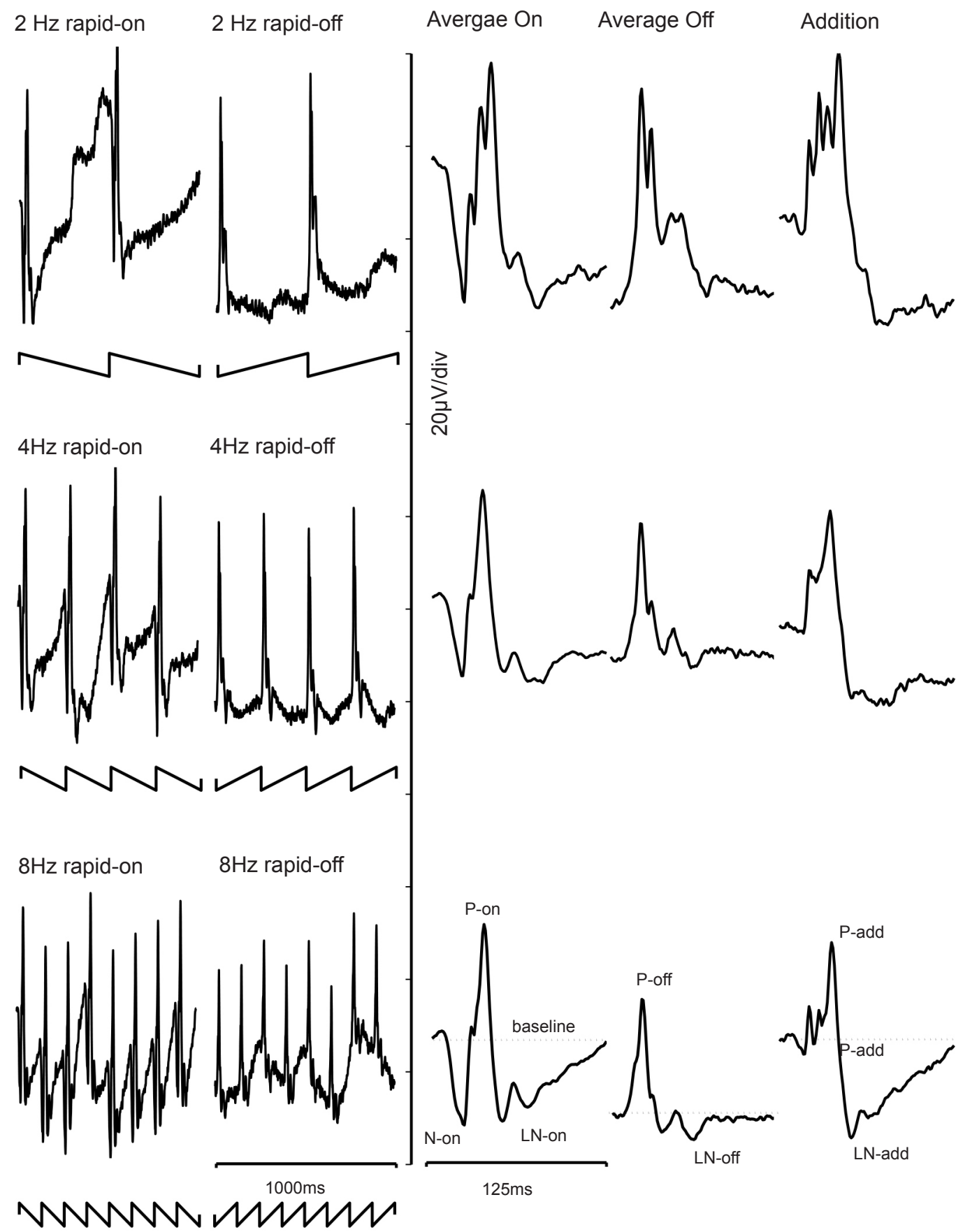

Figure 2. Full-field ERGs elicited using sawtooth stimuli at $2 \mathrm{~Hz}$ (top), $4 \mathrm{~Hz}$ (middle) and $8 \mathrm{~Hz}$ (bottom). The left panel shows some original recordings. Note that the rapid-on stimuli elicit only on-responses and the rapid-off stimuli only off-responses. The third and fourth columns represent the averaged on-response and off-response at respective frequencies. The right column represents the addition of averaged on-and off-responses.

resulting in negative amplitude values. The $\mathrm{LN}$-add was measurable only at lower temporal frequencies and/or higher contrasts.

The right column of Figures 3 and 4 show that the implicit times of on-response components to square wave stimuli decreased significantly with increasing temporal frequency $(\mathrm{N}-o n: \mathrm{p}<0.001$; P-on: $\mathrm{p}<0.001$; LN-on: $\mathrm{p}=0.012$ ) up to $4 \mathrm{~Hz}$ temporal frequency. The implicit times of the off-response components (P-off and LN-off) and the addition components (P-add and $\mathrm{LN}$-add) were not significantly influenced by temporal frequency. Furthermore, the N-on and P-off components had similar implicit times, again indicating that they may have at least partially similar origins. The MANOVA showed that implicit times of the P-on, P-off and P-add components depended significantly on contrast $(\mathrm{N}$ on: $\mathrm{P}<0.001$; P-on: $\mathrm{p}<0.001$; P-off: $\mathrm{p}=0.013$; P-add: $p<0.001$ ), whereas there was no significant effect of 

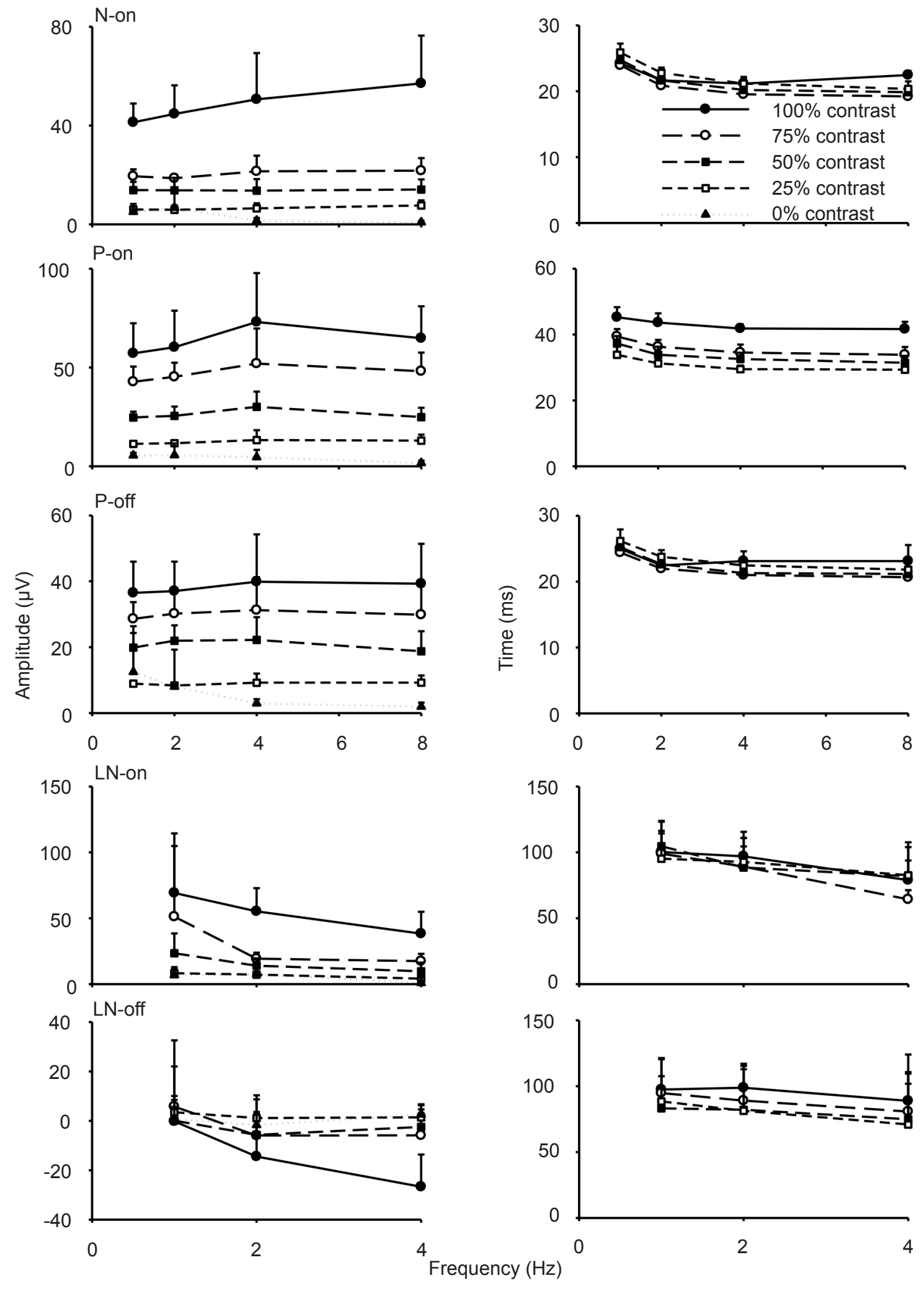

Figure 3. Averaged ( $\pm \mathrm{SD}$ ) amplitudes (left plots) and implicit times (right plots) of different response components (N-on, P-on, P-off, LN-on, and LN-off) elicited by square wave stimuli given as a function of temporal frequency. The results for different stimulus contrasts are shown. The dotted lines in the amplitude plots represent the amplitude of noise measured at $0 \%$ contrast. 

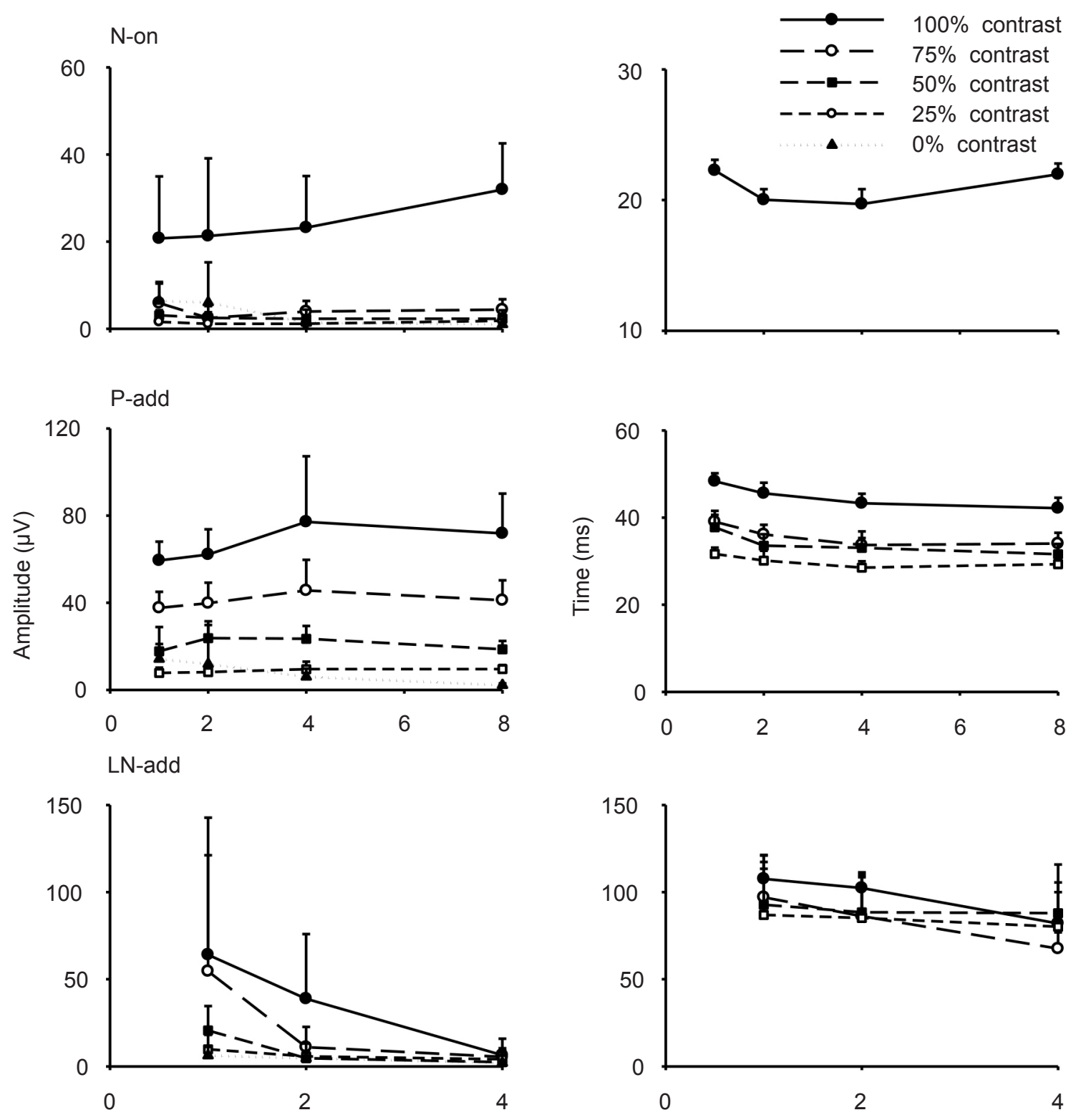

Frequency $(\mathrm{Hz})$

Figure 4. Averaged ( \pm SD) amplitudes (left column) and implicit times (right column) of the components after addition of the on- and off-responses to square wave stimuli ( $\mathrm{N}$-add, $\mathrm{P}$-add and $\mathrm{LN}$-add) plotted as a function of temporal frequency for different stimulus contrasts. The implicit time of $\mathrm{N}$-add component is only shown for the $100 \%$ contrast stimulus because this component was absent with the other stimulus contrasts. The dotted lines in the amplitude plots represent the amplitude of noise measured at $0 \%$ contrast.

contrast on the implicit times of all LNs, which also had similar implicit times.

In contrast to the responses elicited by square wave stimuli, the MANOVA showed that there was a significant influence of temporal frequency on the amplitudes of all response components elicited by sawtooth stimuli except the P-on (N-on: $\mathrm{p}=0.042$; P-off: $\mathrm{p}=0.007$; P-add: $\mathrm{p}<0.001$; LN-on: $\mathrm{p}<0.001$; LN-off: $p=0.019$; LN-add: $p<0.001$; see the left-hand column of Figures 5 and 6). This effect was particularly clear at higher contrasts. No N-add response could be detected (indicating complete cancellation of the $\mathrm{N}$-on and parts of the P-off). In addition, the MANOVA showed a positive relationship between amplitude and contrast for all components ( $\mathrm{N}$-on: $\mathrm{p}<0.001$; P-on: $\mathrm{p}<0.001$; P-off: $\mathrm{p}<0.001$; P-add: $\mathrm{p}<0.001$; LN-on: $\mathrm{p}<0.001$; LN-add: $\mathrm{p}<0.001)$ except for the LN-off. All LN amplitudes were positive, indicating that they were below baseline level.

The implicit times of the components in the responses to sawtooth stimuli are displayed in the right-hand columns of Figures 5 and 6. Temporal frequency had a significant effect on the implicit times of the all components $(\mathrm{N}-\mathrm{on}: \mathrm{p}<0.001$; $\mathrm{P}-\mathrm{on}$ : $\mathrm{p}<0.001$; LN-on: $\mathrm{p}<0.001$; P-off: $\mathrm{p}=0.002$; LN-off: 

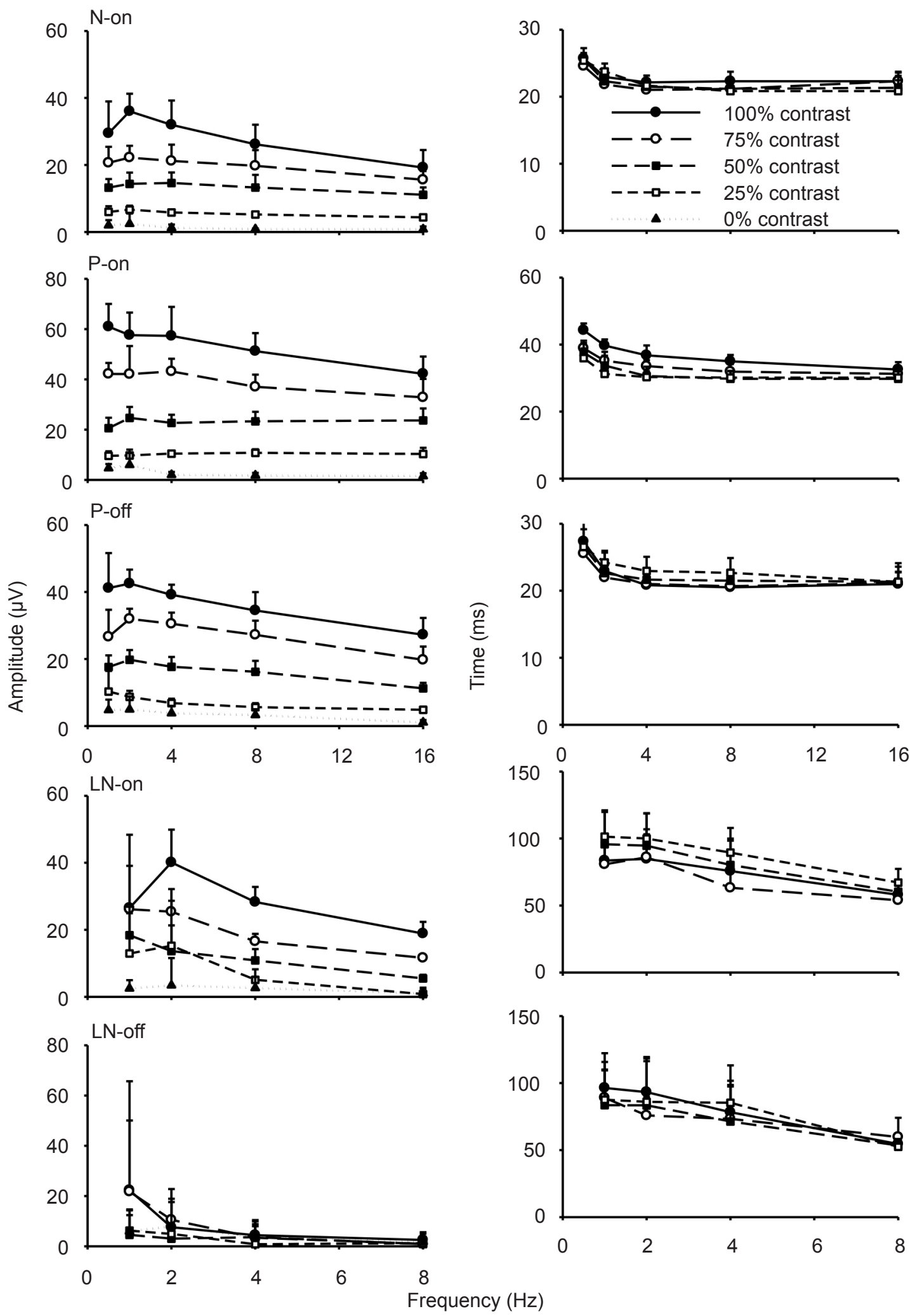

Figure 5. Averaged ( $\pm \mathrm{SD}$ ) amplitudes (left column) and implicit times (right column) of different response components (N-on, P-on, P-off, LN-on, and LN-off) elicited by sawtooth stimuli given as a function of temporal frequency. The results for different stimulus contrasts are shown. The dotted lines in the amplitude plots represent the amplitude of noise measured at $0 \%$ contrast. 


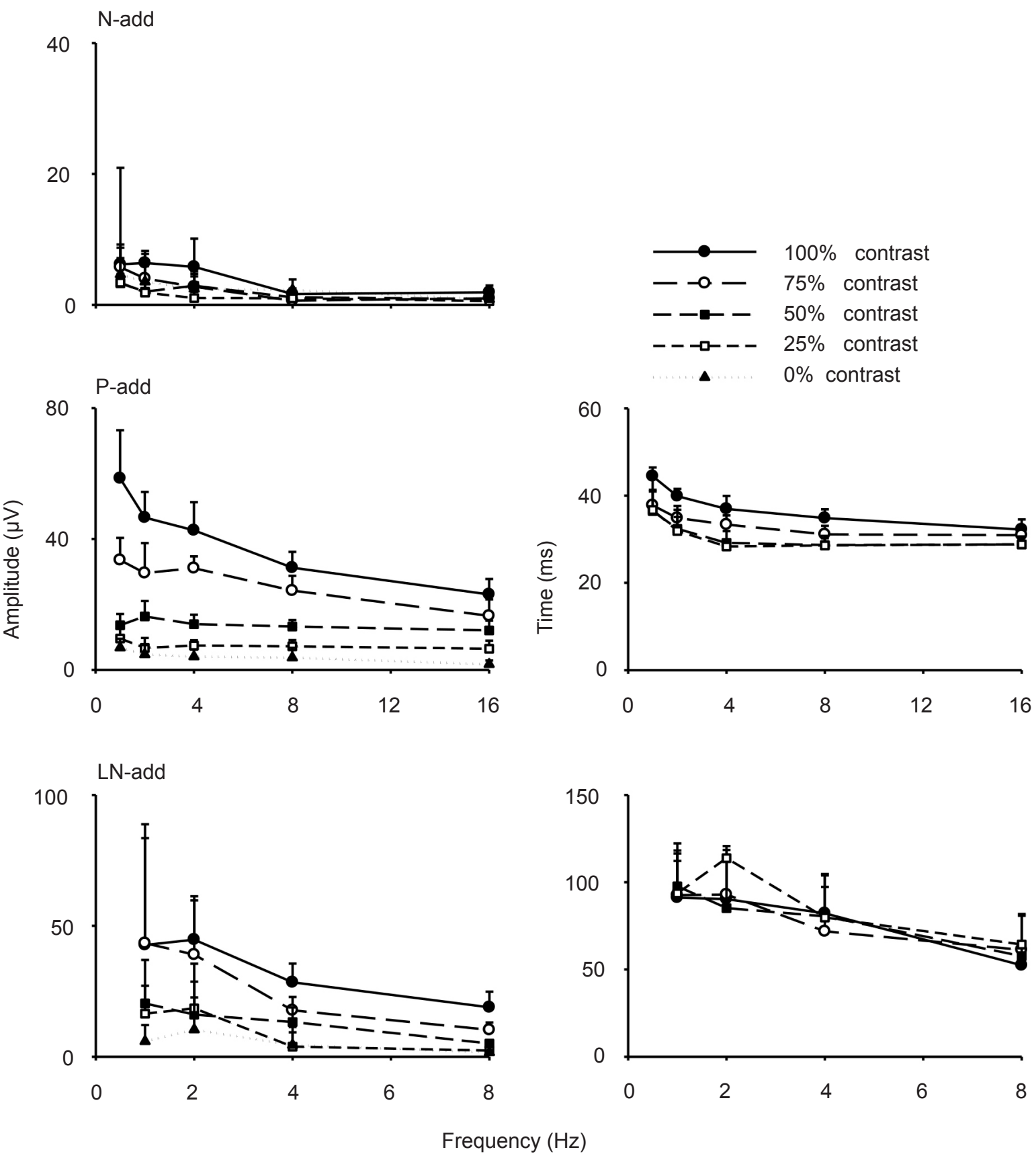

Figure 6. Averaged $( \pm \mathrm{SD})$ amplitudes (left column) and implicit times (right column) of the components after addition of the on- and off-responses to sawtooth stimuli ( $\mathrm{N}$-add, $\mathrm{P}$-add and $\mathrm{LN}$-add) plotted as a function of temporal frequency for different stimulus contrasts. An $\mathrm{N}$-add component was absent at all contrasts so that no implicit times are shown. The dotted lines in the amplitude plots represent the amplitude of noise measured at $0 \%$ contrast.

$\mathrm{p}<0.001$; P-add: $\mathrm{p}<0.001$; LN-add: $\mathrm{p}<0.001$ ). Contrast had a significant effect on the implicit time of $\mathrm{N}$-on $(\mathrm{p}=0.004), \mathrm{P}$-on $(\mathrm{p}<0.001), \mathrm{LN}$-on $(\mathrm{p}<0.001)$ and $\mathrm{P}$-add $(p<0.001)$. Contrast, however, had no significant effect on the implicit times of the P-off, LN-off and LN-add components.

To be able to describe the influence of contrast more directly, the responses to $2 \mathrm{~Hz}$ square wave and $4 \mathrm{~Hz}$ sawtooth stimuli (where the amplitudes of the different components were relatively large with small standard deviations and small noise amplitudes) are plotted in Figure 7 as a function of contrast. The N-on amplitudes (Figure 7, upper left plot) elicited by square wave stimuli show an accelerating nonlinearity at high stimulus contrast, whereas those elicited by sawtooth stimuli increase more linearly with increasing contrast. As mentioned before, the $\mathrm{N}$-add was only present when using $100 \%$ contrast square wave stimuli. The amplitudes of P-on, P-off, P-add and LN-add increased in an approximately linear manner with increasing contrast for both stimulus types. The amplitude of LN-on component elicited by square wave stimuli 

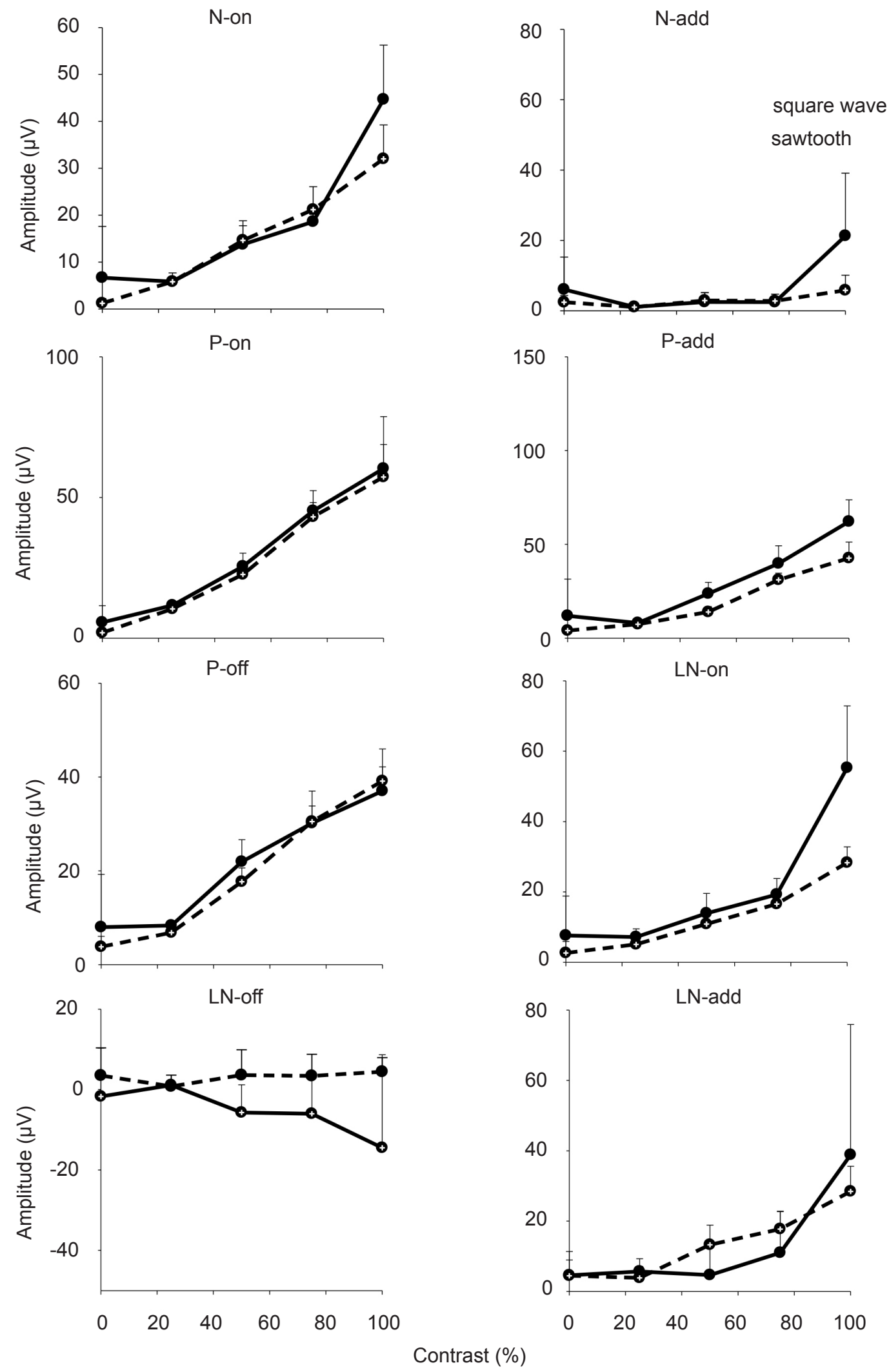

Figure 7. Amplitudes of different response components elicited by square wave (open symbols drawn lines) at $2 \mathrm{~Hz}$ temporal frequency and sawtooth stimuli (closed symbols, dashed lines) at $4 \mathrm{~Hz}$ temporal frequency plotted as a function of contrast. 
Square wave Sawteeth
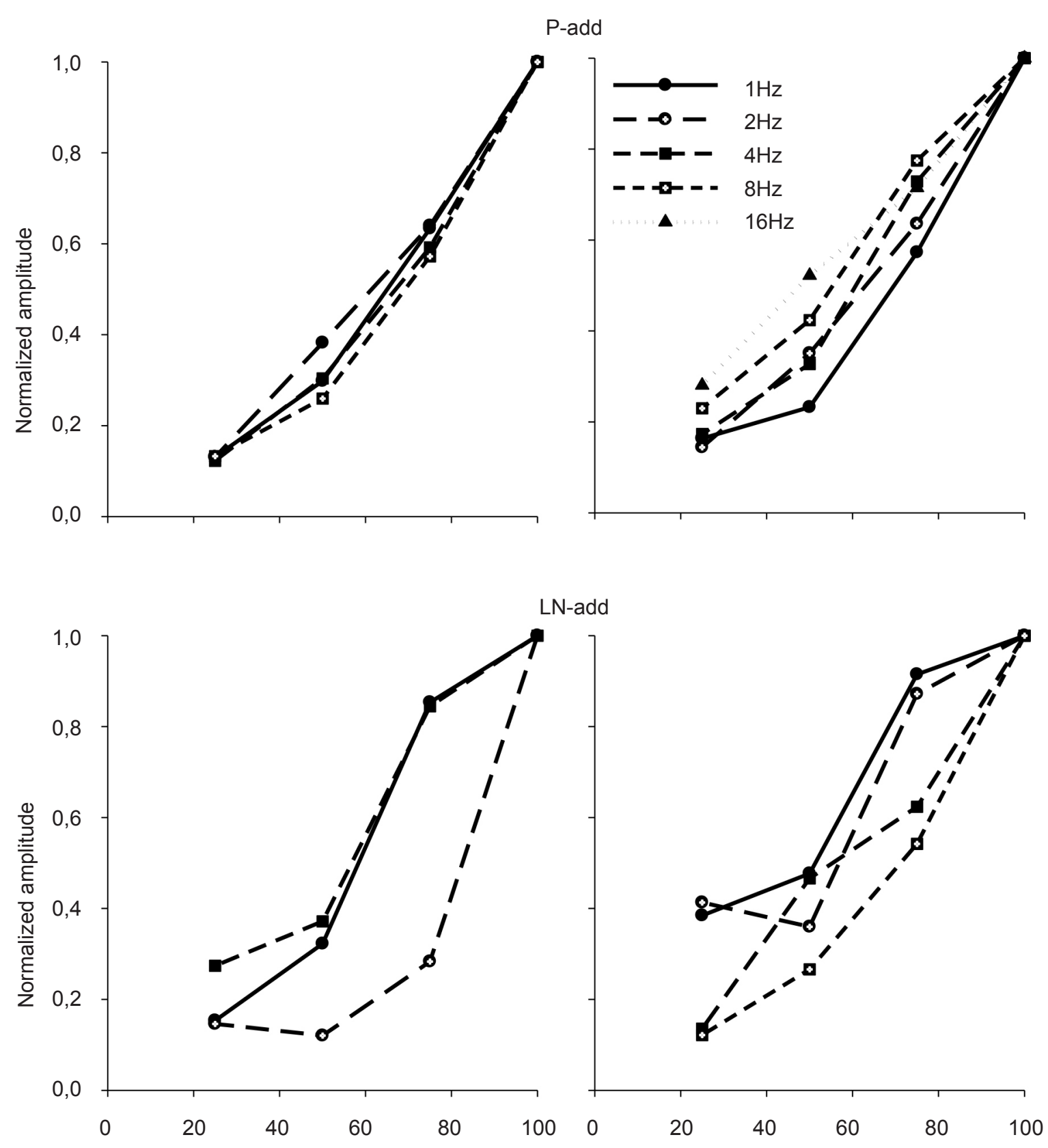

Contrast (\%)

Figure 8. Normalized response amplitudes elicited by square wave (left) and by sawtooth stimuli (right) as a function of stimulus contrast. The amplitudes of the P-add and LN-add components at different temporal frequencies are plotted.

showed accelerating nonlinearity at high contrast. LNoff response waveform elicited by square wave stimuli shows a negative relation with contrast, indicating that the LN-off is above baseline, especially at high contrast.

To obtain a better overview of the influence of contrast at different temporal frequencies, the normalized amplitudes of $\mathrm{P}$-add and $\mathrm{LN}$-add (response asymmetries) at different frequencies are plotted as a function of contrast in Figure 8. Both P-add and LN-add amplitudes show slight accelerating nonlinearities for both stimulus types at all frequencies.

\section{Discussion}

We quantitatively analyzed the ERG response components elicited by square wave and sawtooth stimuli with which the responses of on- and offpathways to the uniform full-field ERG can be separated and investigated. We also studied the effects of stimulus waveform, temporal frequency and contrast on the different components. Furthermore, the asymmetries between the two pathways may indicate responses with inner retinal origins. To our knowledge, such a complete description was lacking to date. As mentioned 
in the Introduction, the PERG is thought to originate in similar response asymmetries. Thus, the asymmetries can possibly be compared with PERG. In addition, the data can be used to choose optimal stimulus conditions for studying the consequences of glaucomatous damage on the different response components. The results of a comparative study between glaucoma patients and age-matched control subjects were published recently (Pangeni, Lammer, Tornow, Horn \& Kremers, 2012). Briefly, the amplitudes of the LN components, particularly the LN-off and the LN-add components, were decreased in patients who also displayed perimetric changes.

The amplitudes and implicit times of the different on- and off-components agree well with those described in previous studies using square wave stimuli (Simpson \& Viswanathan, 2007; Viswanathan et al., 2000) and sawtooth stimuli (Alexander et al., 2001, 2003; Barnes et al., 2002; Khan et al., 2005; Rodrigues et al., 2010) but also to those elicited by long flashes (Shinoda et al., 2001; Sieving, 1993; Sustar et al., 2006). In three studies (Rodrigues et al., 2010; Simpson \& Viswanathan, 2007; Viswanathan et al., 2000), the asymmetries were explicitly investigated in a similar manner as in the present study. Again, the results are qualitatively similar.

\section{Response components and their cellular origins}

The N-on and P-off components elicited by square wave and sawtooth stimuli are partially mirror imaged because they cancel each other out when they are added. This suggests that they have similar cellular origins. In addition, these responses are largely linear and therefore probably originate in the outer retina. Once nonlinearity is revealed, all additional proximal response components will most probably also be influenced by this nonlinearity. It is therefore expected that linear response components will mainly have distal origins. Indeed, there is evidence that both the a- and d-waves in the flash ERG originate in the cone photoreceptor and offbipolar cells (Friedburg, Allen, Mason \& Lamb, 2004; Rangaswamy et al., 2004; Ueno et al., 2006). However, in a different study it was found that the d-wave elicited by sawtooth stimuli contains contributions from both on- and off- bipolar cells (Khan et al., 2005). The results of pharmacological studies show that the b-wave in the flash ERG originates in the on- and off-bipolar cells (Knapp \& Schiller, 1984; Sieving, 1993; Sieving et al., 1994). In the present study, the P-add component did not show significant amplitude and implicit time changes in comparison to the P-on in the on-response, suggesting that the P-add component is mainly determined by the on-response. However, the P-add component has a slightly different waveform than the P-on with an increased number of wavelets (see Figures 1 and 2). This could also be observed in the added responses in a previous study (Rodrigues et al., 2010). The change in waveform morphology of the P-add in the present study is probably partially caused by a complex interaction between the P-on, P-off and both LN components.
The late negative components (LN-on and LN-off) are probably homologues of the photopic negative response (PhNR) of the flash ERG that originates from the retinal ganglion cells (Rangaswamy, Digby, Harwerth \& Frishman, 2005; Viswanathan et al., 1999, 2000). The PhNR in the flash ERG are probably overlapping LN-on and LN-off responses (Kondo et al., 2008). We found that the LN-add amplitude is slightly increased compared to either LN-on or LN-off in both square wave and sawtooth stimuli, suggesting some asymmetry in those late negative components. This was also found by Rodrigues et al. (2010) in the multifocal ERG and makes the LN-add interesting for the diagnosis of inner retinal disorders.

\section{The effects of contrast, temporal frequency and stimulus waveform}

The amplitudes of all components increased with increasing contrast (Figures 3-6). This is not surprising and has been observed several times in the human fullfield flicker ERG using sine-wave stimuli (Burns, Elsner \& Kreitz, 1992; Odom, Reits, Burgers \& Riemslag, 1992; Pangeni, Horn \& Kremers, 2010) and flashes (Kondo et al., 2000; Peachey, Alexander, Fishman \& Derlacki, 1989; Wali \& Leguire, 1992). Furthermore, response amplitudes of single ganglion cells to square wave and sawtooth stimuli increased with increasing contrast (Kremers, Lee, Pokorny \& Smith, 1993).

We found that the implicit time of the P-on to both stimuli increased with increasing contrast. This is in agreement with previous data on the b-wave implicit time in the flash ERG (Kondo et al., 2000; Peachey et al., 1989; Wali \& Leguire, 1992), although the implicit time of the b-wave in the long-flash ERG did not change (Kondo et al., 2000). The latency of single retinal cell responses decrease with increasing contrast (Lee, Pokorny, Smith \& Kremers, 1994). The P-on component, however, originates most probably in activity of bipolar cells. It is not known how the responses of primate bipolar cells depend on stimulus contrast. Furthermore, possibly more cells are recruited when contrast is increased. It is not known how this affects the response latency.

The implicit time of the LN-on response to sawtooth stimuli decreased slightly but significantly with increasing contrast, suggesting a different cellular origin than the P-on. Indeed, the PhNR in the flash ERG probably originates in spiking activity of retinal ganglion cells (Viswanathan et al., 1999, 2000) whose response phases advance with increasing contrasts owing to a gain control mechanism (Benardete, Kaplan \& Knight, 1992; Lee et al., 2000; Shapley \& Victor, 1979; Yeh, Lee \& Kremers, 1995). Possibly, such contrast gain control mechanisms are not present in the activity of more distal cell types.

The amplitudes of all LNs decreased with increasing temporal frequency using square wave and sawtooth stimuli. This is in qualitative agreement with responses shown by Alexander et al. (2001) and Khan et al. 
(2005). Single retinal ganglion cell responses showed an optimal temporal frequency at about $20 \mathrm{~Hz}$ to these stimuli (Kremers et al., 1993), indicating that there is no direct relationship between single ganglion cell data and the LNs.

Qualitatively, sawtooth and square-wave stimuli provide similar results. Square wave stimuli may be more efficient because on- and off-responses can be measured in one cycle. However, the on- and offresponses to square wave stimuli merge at lower temporal frequencies than for the sawtooth stimuli. Therefore, sawtooth stimuli can offer a more complete picture about the effects of temporal frequency. In addition, at low temporal frequencies, adaptation processes may play a larger role with square wave stimuli.

The data provide a quantitative description of the different responses with which future data (for instance obtained in different patient groups or after isolating the responses driven by single photoreceptor types) can be compared. Based on the present data, we suggest that responses to sawtooth stimuli are superior to those elicited by square wave stimuli because onand off-responses were clearly separated and because adaptation effects (resulting in more nonlinear contrast dependence; see Figure 7) are expected to be smaller.

\section{Comparison of response additions with the PERG}

The data can also be compared quantitatively with other response data where similar mechanisms are assumed to play a role (such as the PERG). In the present study, the response asymmetries in the uniform field ERG are extracted by adding on- and off-responses. Very similar asymmetries are thought to be the origin of the PERG (Baker \& Hess, 1984; Riemslag et al., 1985; Spekreijse et al., 1973). Indeed, Simpson \& Viswanathan (2007) and Viswanathan et al. (2000) simulated the transient PERG by summing the ERG responses to light increments and decrements of the uniform field ERG. Rodrigues et al. (2010) performed a similar analysis to describe the response asymmetries using sawtooth stimuli in multifocal ERG. The P46add and N100add components in their study are similar to the P-add and LN-add components in the present study. If the response additions are indeed comparable with the PERGs, then the P-add and the LN-add are comparable to the P50 and N95 of the PERG, respectively. In agreement with this notion, the relationship between the PERG amplitudes and contrast (Ben-Shlomo, Bach \& Ofri, 2007; Hess \& Baker, 1984; Korth \& Rix, 1985; Thompson \& Drasdo, 1989; Zapf \& Bach, 1999) is similar to the one between the full field response additions and contrast as shown in the present study.

However, edge effects and lateral interactions may result in differences between the full field additions and the PERG. The PERG response depends on spatial frequency of the stimulus, indicating that lateral interactions cannot be neglected. This means that the full field asymmetries are comparable to PERGs measured with large check sizes. Indeed, Viswanathan et al.
(2000) found that the difference between the simulation and the actual PERG increases with increasing spatial frequency of the PERG. However, a direct comparison between response asymmetries and PERG data obtained in identical individuals and very similar conditions are needed to establish a closer comparison.

\section{Acknowledgements}

The work was supported by the German Research Council (DFG) grant KR 1317/9-1. JK is a Fellow in the Excellence Program of the Hertie Foundation. The authors thank Prof. Kruse for general support and Silvia Rühl, Anja Ehrhardt and Astrid Kraus for technical support.

\section{References}

Alexander, K. R., Barnes, C. S. \& Fishman, G. A. (2003). ON-pathway dysfunction and timing properties of the flicker ERG in carriers of X-linked retinitis pigmentosa. Investigative Ophthalmology \& Visual Science, 44, 4017-4025.

Alexander, K. R., Fishman, G. A., Barnes, C. S. \& Grover, S. (2001). ON-response deficit in the electroretinogram of the cone system in X-linked retinoschisis. Investigative Ophthalmology \& Visual Science, 42, 453-459.

Bach, M. \& Hoffmann, M. B. (2008). Update on the pattern electroretinogram in glaucoma. Optometry and Vision Science, 85 (6), 386-395.

Bach, M., Unsoeld, A. S., Philippin, H., Staubach, F., Maier, P., Walter, H. S., Bomer, T. G. \& Funk, J. (2006). Pattern ERG as an early glaucoma indicator in ocular hypertension: A long-term, prospective study. Investigative Ophthalmology \& Visual Science, 47 (11), 4881-4887.

Baker, C. L., Jr. \& Hess, R. F. (1984). Linear and nonlinear components of human electroretinogram. Journal of Neurophysiology, 51 (5), 952-967.

Barnes, C. S., Alexander, K. R. \& Fishman, G. A. (2002). A distinctive form of congenital stationary night blindness with cone ONpathway dysfunction. Ophthalmology, 109, 575-583.

Ben-Shlomo, G., Bach, M. \& Ofri, R. (2007). Temporal and spatial frequencies interact in the contrast transfer function of the pattern electroretinogram. Vision Reseach, 47 (15), 1992-1999.

Benardete, E. A., Kaplan, E. \& Knight, B. W. (1992). Contrast gain control in the primate retina: $\mathrm{P}$ cells are not X-like, some $\mathrm{M}$ cells are. Visual Neuroscience, 8, 483-486.

Biersdorf, W. R. (1968). Rod and cone contributions to the off-effect of the human ERG. Investigative Ophthalmology, 7 (4), 371-377.

Bloomfield, S. A. (1996). Effect of spike blockade on the receptivefield size of amacrine and ganglion cells in the rabbit retina. Journal of Neurophysiology, 75 (5), 1878-1893.

Burns, S. A., Elsner, A. E. \& Kreitz, M. R. (1992). Analysis of nonlinearities in the flicker ERG. Optometry and Vision Science, 69, 95-105.

Bush, R. A. \& Sieving, P. A. (1996). Inner retinal contributions to the primate photopic fast flicker electroretinogram. Journal of the Optical Society of America A, 13 (3), 557-565.

Dryja, T. P., McGee, T. L., Berson, E. L., Fishman, G. A., Sanberg, M. A., Alexander, K. R., ... Rajagopalan, A. S. (2005). Night blindness and abnormal cone electroretinogram $\mathrm{ON}$ responses in patients with mutations in the GRM6 gene encoding mGluR6. Proceedings of the National Academy of Science of the United Sates of America, 102 (12), 4884-4889.

Evers, H. U. \& Gouras, P. (1986). Three cone mechanisms in the primate electroretinogram: two with, one without off-center bipolar responses. Vision Research, 26 (2), 245-254.

Friedburg, C., Allen, C. P., Mason, P. J. \& Lamb, T. D. (2004). Contribution of cone photoreceptors and post-receptoral mechanisms to the human photopic electroretinogram. Journal of Physiology, 556, 819-834.

Hess, R. F. \& Baker, C. L., Jr. (1984). Human pattern-evoked electroretinogram. Journal of Neurophysiology, 51 (5), 939-951. 
Howarth, C. I. (1961). On-off interaction in the human electroretinogram. Journal of the Optometry Society of America, $51,345-352$.

Khan, N. W., Kondo, M., Hiriyanna, K. T., Jamison, J. A., Bush, R. A. \& Sieving, P. A. (2005). Primate retinal signaling pathways: Suppressing ON-pathway activity in monkey with glutamate analogues mimics human CSNB1-NYX genetic night blindness. Journal of Neurophysiology, 93 (1), 481-492.

Knapp, A. G. \& Schiller, P. H. (1984). The contribution of on-bipolar cells to the electroretinogram of rabbits and monkeys. A study using 2-amino-4-phosphonobutyrate (APB). Vision Research, 24 (12), 1841-1846.

Kondo, M., Kurimoto, Y., Sakai, T., Koyasu, T., Miyata, K., Ueno, S. \& Terasaki, H. (2008). Recording focal macular photopic negative response (PhNR) from monkeys. Investigative Ophthalmology \& Visual Science, 49 (8), 3544-3550.

Kondo, M. \& Miyake, Y. (2000). Assessment of local cone on- and off-pathway function using multifocal ERG technique. Documenta Ophthalmologica, 100, 139-154.

Kondo, M., Piao, C. H., Tanikawa, A., Horiguchi, M., Terasaki, H. \& Miyake, Y. (2000). Amplitude decrease of photopic ERG $\mathrm{b}$-wave at higher stimulus intensities in humans. Japan Journal of Ophthalmology 44 (1), 20-28.

Korth, M. \& Rix, R. (1985). Changes in spatial selectivity of patternERG components with stimulus contrast. Graefes Archives of Clinical and Experimental Ophthalmology, 223 (1), 23-28.

Kremers, J., Lee, B. B., Pokorny, J. \& Smith, V. C. (1993). Responses of macaque ganglion cells and human observers to compound periodic waveforms. Vision Research, 33, 1997-2011.

Lee, B. B., Pokorny, J., Smith, V. C. \& Kremers, J. (1994). Responses to pulses and sinusoids in macaque ganglion cells. Vision Research, 34 (23), 3081-3095.

Lee, B. B., Silveira, L. C. L., Yamada, E. S., Hunt, D. M., Kremers, J., Martin, P. R., Troy, J. B. \& da Silva-Filho, M. (2000). Visual responses of ganglion cells of a New-World primate, the capuchin monkey, Cebus apella. Journal of Physiology, 528 (3), 573-590.

North, R. V., Jones, A. L., Drasdo, N., Wild, J. M. \& Morgan, J. E. (2010). Electrophysiological evidence of early functional damage in glaucoma and ocular hypertension. Investigative Ophthalmology \& Visual Science, 51 (2), 1216-1222.

Odom, J. V., Reits, D., Burgers, N. \& Riemslag, F. C. (1992). Flicker electroretinograms: A systems analytic approach. Optometry and Visual Science, 69 (2), 106-116.

Pangeni, G., Horn, F. K. \& Kremers, J. (2010). A new interpretation of components in the ERG signals to sine wave luminance stimuli at different temporal frequencies and contrasts. Visual Neuroscience, 27 (3-4), 79-90.

Pangeni, G., Lammer, R., Tornow, R. P., Horn, F. K. \& Kremers, J. (2012). On- and off-response ERGs elicited by sawtooth stimuli in normal subjects and glaucoma patients. Documenta Ophthalmologica, 124 (3), 237-248.

Peachey, N. S., Alexander, K. R., Fishman, G. A. \& Derlacki, D. J. (1989). Properties of the human cone system electroretinogram during light adaptation. Applied Optometry, 28 (6), 1145-1150.

Rangaswamy, N. V., Digby, B., Harwerth, R. S. \& Frishman, L. J. (2005). Optimizing the spectral characteristics of a Ganzfeld stimulus used for eliciting the photopic negative response (PhNR). Investigative Ophthalmology \& Visual Science (Supplement), 46, \#4762.

Rangaswamy, N. V., Frishman, L. J., Dorotheo, E. U., Schiffman, J. S., Bahrani, H. M. \& Tang, R. A. (2004). Photopic ERGs in patients with optic neuropathies: Comparison with primate ERGs after pharmacologic blockade of inner retina. Investigative Ophthalmology \& Visual Science, 45, 3827-3837.

Riemslag, F. C., Ringo, J. L., Spekreijse, H. \& Verduyn Lunel, H. F. (1985). The luminance origin of the pattern electroretinogram in man. Journal of Physiology, 363, 191-209.

Rodrigues, A. R., Filho Mda, S., Silveira, L. C. \& Kremers, J. (2010). Spatial distributions of on- and off-responses determined with the multifocal ERG. Documenta Ophthalmologica, 120 (2), 145-158.

Shapley, R. M. \& Victor, J. D. (1979). Nonlinear spatial summation and the contrast gain control of cat retinal ganglion cells. Journal of Physiology, 290, 141-161.

Shinoda, K., Ohde, H., Mashima, Y., Inoue, R., Ishida, S., Inoue, M., ... Oguchi, Y. (2001). On- and off-responses of the photopic electroretinograms in X-linked juvenile retinoschisis. American Journal of Ophthalmology, 131 (4), 489-494.

Sieving, P. A. (1993). Photopic on- and off-pathway abnormalities in retinal dystrophies. Transactions of the American Ophthalmological Society, 91, 701-773.

Sieving, P. A., Murayama, K. \& Naarendorp, F. (1994). Pushpull model of the primate photopic electroretinogram: A role for hyperpolarizing neurons in shaping the b-wave. Visual Neuroscience, 11 (3), 519-532.

Simpson, M. \& Viswanathan, S. (2007). Comparison of uniform field and pattern electroretinograms of humans. Journal of Modern Optics, 54, 1281-1288.

Spekreijse, H., van der Tweel, H. \& Zuidema, T. (1973). Contrast evoked responses in man. Vision Research, 13, 1577-1601.

Stafford, D. K. \& Dacey, D. M. (1997). Physiology of the A1 amacrine: A spiking, axon-bearing interneuron of the macaque monkey retina. Visual Neuroscience, 14 (3), 507-522.

Sustar, M., Hawlina, M. \& Brecelj, J. (2006). ON- and OFFresponse of the photopic electroretinogram in relation to stimulus characteristics. Documenta Ophthalmologica, 113, 43-52.

Thompson, D. \& Drasdo, N. (1989). The effect of stimulus contrast on the latency and amplitude of the pattern electroretinogram. Vision Research, 29 (3), 309-313.

Ueno, S., Kondo, M., Ueno, M., Miyata, K., Terasaki, H. \& Miyake, Y. (2006). Contribution of retinal neurons to d-wave of primate photopic electroretinograms. Vision Research, 46, 658-664.

Viswanathan, S., Frishman, L. J. \& Robson, J. G. (2000). The uniform field and pattern ERG in macaques with experimental glaucoma: Removal of spiking activity. Investigative Ophthalmology \& Visual Science, 41 (9), 2797-2810.

Viswanathan, S., Frishman, L. J., Robson, J. G., Harwerth, R. S. $\&$ Smith, E. L. $3^{\text {rd }}$. (1999). The photopic negative response of the macaque electroretinogram: reduction by experimental glaucoma. Investigative Ophthalmology \& Visual Science, 40 (6), 1124-1136.

Wali, N. \& Leguire, L. E. (1992). The photopic hill: A new phenomenon of the light adapted electroretinogram. Documenta Ophthalmologica, 80 (4), 335-345.

Yeh, T., Lee, B. B. \& Kremers, J. (1995). Temporal response of ganglion cells of the macaque retina to cone-specific modulation. Journal of the Optical Society of America A, 12 (3), 456-464.

Zapf, H. R. \& Bach, M. (1999). The contrast characteristic of the pattern electroretinogram depends on temporal frequency. Graefes Archives of Clinical and Expermental Ophthalmology, 237 (2), 9399. 